\title{
Newton-Euler Dynamic Equations of Motion for a Multi-body Spacecraft
}

\author{
Eric Stoneking * \\ NASA Goddard Space Flight Center, Greenbelt, MD 20771, USA
}

\begin{abstract}
The Magnetospheric MultiScale (MMS) mission employs a formation of spinning spacecraft with several flexible appendages and thruster-based control. To understand the complex dynamic interaction of thruster actuation, appendage motion, and spin dynamics, each spacecraft is modeled as a tree of rigid bodies connected by spherical or gimballed joints. The method presented facilitates assembling by inspection the exact, nonlinear dynamic equations of motion for a multibody spacecraft suitable for solution by numerical integration. The building block equations are derived by applying Newton's and Euler's equations of motion to an "element" consisting of two bodies and one joint (spherical and gimballed joints are considered separately). Patterns in the "mass" and "force" matrices guide assembly by inspection of a general N-body tree-topology system. Straightforward linear algebra operations are employed to eliminate extraneous constraint equations, resulting in a minimum-dimension system of equations to solve. This method thus combines a straightforward, easily-extendable, easily-mechanized formulation with an efficient computer implementation.
\end{abstract}

\section{Introduction}

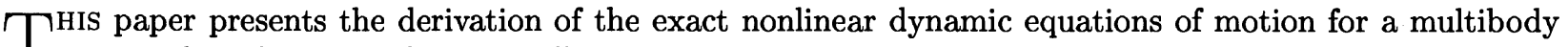
1 spacecraft with spherical or gimballed joints, suitable for computer solution. The formulation presented in this paper is motivated by the Magnetospheric MultiScale (MMS) mission, currently in mission definition at Goddard Space Flight Center. MMS will employ a formation of four spacecraft in a highly elliptical orbit to study the Earth's magnetosphere. Each spacecraft is composed of a cylindrical central body, four "rigid" instrument booms, and four 50-meter wire booms. The spacecraft are spin-stabilized, and require periodic thruster operation for both formation maintenance and large orbital maneuvers. To study the interaction between thruster forces, spin dynamics, and boom flexibility, a simulation tool with sufficient fidelity is essential. To keep the model conceptually simple, we choose to model the booms (both rigid and wire) as chains of rigid bodies, with elastic and damping properties concentrated at the joints. To confirm the validity of this construction, we compare the simulated motion of one-, two-, and three-segment boom models; in the limit, the results should converge to that of the continuous structure. Seeking a dynamical formulation which allows easy adaptation from one-segment booms to two- and three-segment booms leads us to the general method presented here.

The goal of this paper is to develop and describe algorithms in sufficient detail that the reader may, given properties of a multi-body system, assemble by inspection exact, nonlinear dynamical equations of motion suitable for numerical integration by computer. While the literature is rich with multi-degree-of-freedom dynamic formulations, most have theoretical or practical limitations which compromise their utility for problems like the MMS spacecraft. Finite-element models allow straightforward modelling of many modes; the method here presented borrows from finite-element methods in the assembly of large systems from small building blocks. Finite-element models, however, often linearize the equations of motion early in the process, and are consequently best suited to small perturbations from some nominal motion. Nonlinear coupling may be important to the MMS dynamics, and so a method was sought to preserve it. Hand derivation of nonlinear equations of motion using, for example, Kane's or Lagrange's methods, rapidly becomes laborious as the number of bodies grows. Computer aids help (Autolev, for example, even outputs source code), but

\footnotetext{
*Aerospace Engineer, Code 591, AIAA Member.
} 


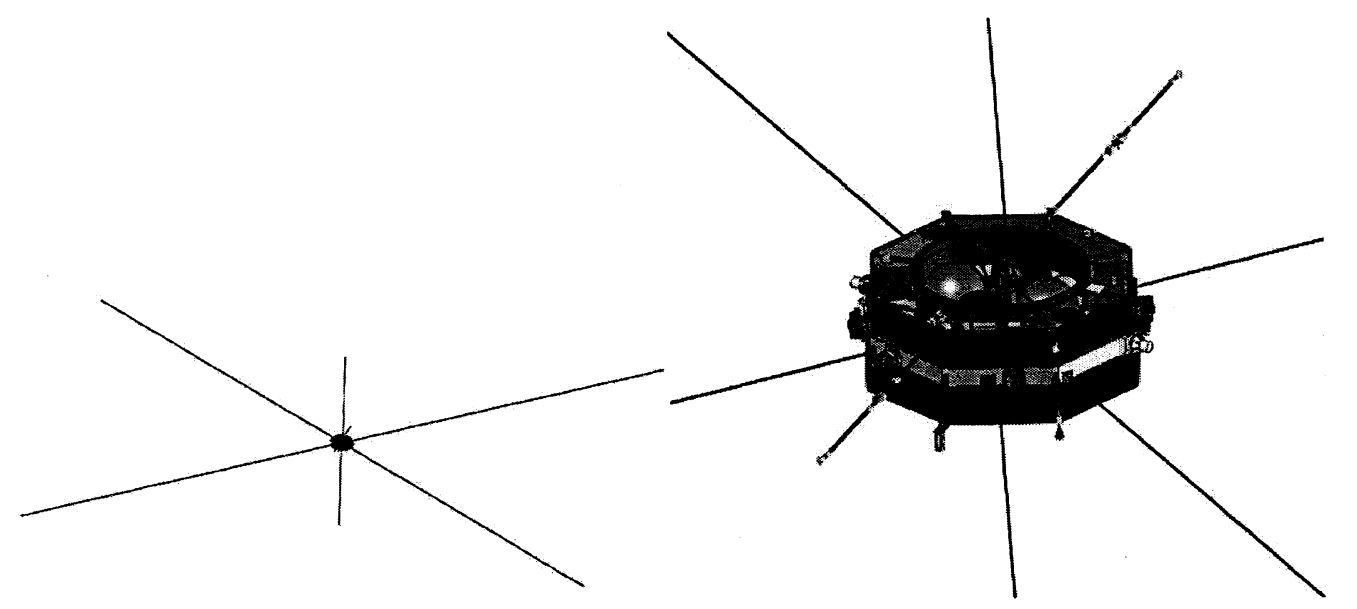

Figure 1. MMS Spacecraft, Scale and Closeup

each model change (say from a one-segment boom to a three-segment boom) requires a new dynamic model to be linked to the simulation. The formulation presented in this paper allows the model information to be provided without recompilation of the simulation code. This point is exemplified by the simulation results presented, in which multiple spacecraft, with distinct dynamical models, are simulated concurrently using the same software functions.

\section{Equations of Motion for Two Bodies and One Spherical Joint}

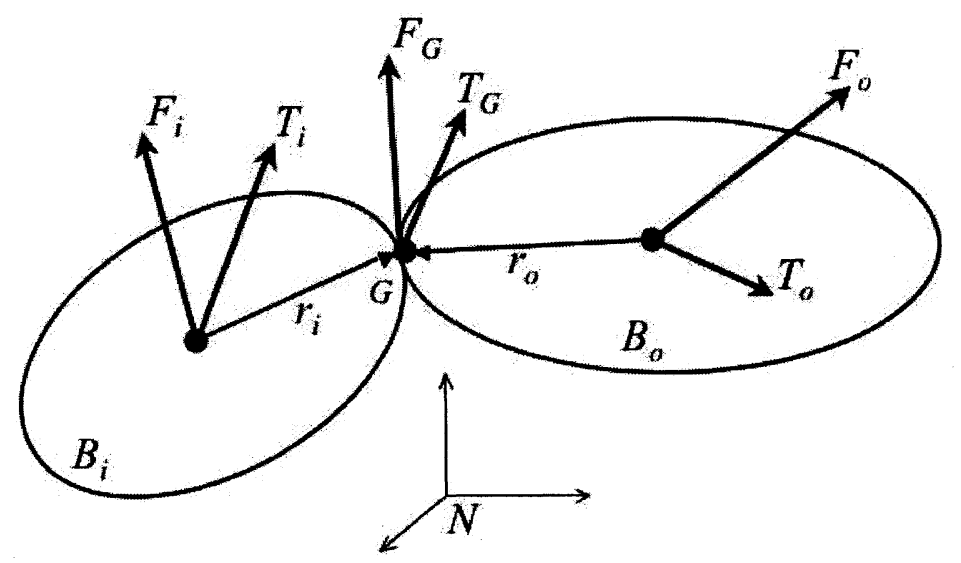

Figure 2. Two Bodies Connected by a Spherical Joint

Translational motion is governed by Newton's second law, which we may write:

$$
m \dot{v}=F
$$

The rotational motion of a rigid body is governed by Euler's equation:, 2

$$
I \dot{\omega}=T-\omega \times H
$$

where the overdot operator, $(\dot{)})$, denotes differentiaton with respect to time in a "local" frame; time derivatives of vectors in a frame $A$ are related to derivatives in an inertial frame $N$ by

$$
{ }^{N} \frac{d}{d t}(v)={ }^{A} \frac{d}{d t}(v)+{ }^{N} \omega^{A} \times v=\dot{v}+{ }^{N} \omega^{A} \times v
$$


For translational quantities, the most convenient "local" frame is the $N$ frame itself.

Consider the simplest possible multibody case, depicted in figure 2: an "inner" body, $B_{i}$, and an "outer" body, $B_{o}$, connected by a spherical joint, $G$. We seek the equations of motion for attitude and translation of the two bodies with respect to an inertial reference frame, $N$. Considering each body separately, and explicitly separating the joint constraint force and torque from all other applied forces and torques, we may write Euler's and Newton's equations of motion as:

$$
\begin{aligned}
I_{i} \dot{\omega}_{i} & =T_{i}-\omega_{i} \times H_{i}+T_{G}+r_{i} \times F_{G} \\
I_{o} \dot{\omega}_{o} & =T_{o}-\omega_{o} \times H_{o}-T_{G}-r_{o} \times F_{G} \\
m_{i} \dot{v}_{i} & =F_{i}+F_{G} \\
m_{o} \dot{v}_{o} & =F_{o}-F_{G}
\end{aligned}
$$

where the symbols used for each body are listed in table 1 , and where $F_{G}$ and $T_{G}$ are the constraint force

Table 1. Symbols Used in Newton-Euler Equations

\begin{tabular}{|c|c|}
\hline Symbol & Description \\
\hline \hline$\omega_{i}, \omega_{o}$ & Angular velocity \\
\hline$v_{i}, v_{o}$ & Velocity of mass center \\
\hline$I_{i}, I_{o}$ & Central moments of inertia \\
\hline$m_{i}, m_{o}$ & Mass \\
\hline$r_{i}, r_{o}$ & Vector from mass center to joint \\
\hline$H_{i}, H_{o}$ & Central angular momentum \\
\hline$F_{i}, F_{o}$ & Resultant external force \\
\hline$T_{i}, T_{o}$ & Resultant external torque \\
\hline
\end{tabular}

and torque at the joint. The local frames for differentiation of $\omega_{i}$ and $\omega_{o}$ are the frames fixed in $B_{i}$ and $B_{o}$, respectively; for both $v_{i}$ and $v_{o}$, the $N$ frame is the local frame. For a spherical joint, the constraint torque represents the material elasticity and damping lumped in the joint, as well as any active control torques; the constitutive relations to compute $T_{G}$ are assumed to be provided by the user. The constraint force, $F_{G}$, is an unknown to be eliminated. We arbitrarily choose the signs of $F_{G}$ and $T_{G}$ to act in a positive sense on $B_{i}$, and in a negative sense on $B_{o}$. In the absence of momentum storage devices, $H=I \omega$. For completeness, the addition of embedded momentum wheels is addressed in the Appendix.

The joint constraint is introduced by equating two expressions for the velocity of $G$ in $N$ :

$$
v_{G}=v_{i}+\omega_{i} \times r_{i}=v_{o}+\omega_{o} \times r_{o}
$$

Differentiating with respect to time (in $N$ ) yields the constraint equation:

$$
\dot{v}_{i}+\dot{\omega}_{i} \times r_{i}+\omega_{i} \times\left(\omega_{i} \times r_{i}\right)=\dot{v}_{o}+\dot{\omega}_{o} \times r_{o}+\omega_{o} \times\left(\omega_{o} \times r_{o}\right)
$$

Equations (1)-(5) are five vector equations in the five vector unknowns $\dot{\omega}_{i}, \dot{\omega}_{o}, \dot{v}_{i}, \dot{v}_{o}$, and $F_{G}$.

For compactness, we introduce two notational devices. The over-tilde operator, (), denotes a 3-by-3 skew-symmetric matrix so that $\tilde{x} y=x \times y$. Similarly, the overbar operator, $\overline{()}$, denotes a symmetric matrix such that $\bar{x} y=x \times(x \times y)$. If the scalar components of the vector $x$ are $\left(x_{1}, x_{2}, x_{3}\right)$, then

$$
\tilde{x}=\left[\begin{array}{ccc}
0 & -x_{3} & x_{2} \\
x_{3} & 0 & -x_{1} \\
-x_{2} & x_{1} & 0
\end{array}\right], \quad \bar{x}=\left[\begin{array}{ccc}
-x_{2}^{2}-x_{3}^{2} & x_{1} x_{2} & x_{1} x_{3} \\
x_{2} x_{1} & -x_{3}^{2}-x_{1}^{2} & x_{2} x_{3} \\
x_{3} x_{1} & x_{3} x_{2} & -x_{1}^{2}-x_{2}^{2}
\end{array}\right]
$$

Note that $-\tilde{x}=x^{T}$. 
Now we write equations (1)-(5) in matrix form:

$$
\left[\begin{array}{ccccc}
I_{i} & 0 & 0 & 0 & -\tilde{r}_{i} \\
0 & I_{o} & 0 & 0 & \tilde{r}_{o} \\
0 & 0 & m_{i} \mathbf{1} & 0 & -\mathbf{1} \\
0 & 0 & 0 & m_{o} \mathbf{1} & \mathbf{1} \\
-\tilde{r}_{i}^{T} & \tilde{r}_{o}^{T} & -\mathbf{1} & \mathbf{1} & 0
\end{array}\right]\left\{\begin{array}{c}
\dot{\omega}_{i} \\
\dot{\omega}_{o} \\
\dot{v}_{i} \\
\dot{v}_{o} \\
F_{G}
\end{array}\right\}=\left\{\begin{array}{c}
T_{i}-\tilde{\omega}_{i} H_{i}+T_{G} \\
T_{o}-\tilde{\omega}_{o} H_{o}-T_{G} \\
F_{i} \\
F_{o} \\
\bar{\omega}_{i} r_{i}-\bar{\omega}_{o} r_{o}
\end{array}\right\}
$$

where 1 denotes the 3-by-3 identity matrix.

Table 2. Reference Frames for Expression of Components of Dynamic Entities

\begin{tabular}{|c|c|c|}
\hline Items Expressed in $B_{i}$ & Items Expressed in $B_{o}$ & Items Expressed in $N$ \\
\hline \hline$I_{i}$ & $I_{o}$ & $v_{i}, \dot{v}_{i}$ \\
$r_{i}, \tilde{r}_{i}$ & $r_{o}, \tilde{r}_{o}$ & $v_{o}, \dot{v}_{o}$ \\
$\dot{\omega}_{i}, \tilde{\omega}_{i}, \tilde{\omega}_{i}, \bar{\omega}_{i}$ & $\omega_{o}, \dot{\omega}_{o}, \tilde{\omega}_{o}, \bar{\omega}_{o}$ & $F_{i}$ \\
$T_{i}$ & $T_{o}$ & $F_{o}$ \\
$H_{i}$ & $H_{o}$ & $F_{G}$ \\
$T_{G}$ & & \\
\hline
\end{tabular}

These equations of motion are basis-free. To solve them numerically, all items in an equation must be expressed in a common reference frame. Table 2 lists dynamic entities of interest and the frames in which they are naturally expressed. Suppose, for example, that a thruster is mounted on $B_{i}$. The force it exerts on $B_{i}$ is expressed in the $N$ frame for addition into $F_{i}$, while the torque it exerts on $B_{i}$ is expressed in $B_{i}$, as it must be added into $T_{i}$. It proves most convenient to express the first row of equation (6) in $B_{i}$, the second in $B_{o}$, and the remaining three in $N$. The choice to express $T_{G}$ in $B_{i}$ rather than in $B_{o}$ is arbitrary.

The transformation between reference frames $\mathrm{X}$ and $\mathrm{Y}$ is represented by the direction cosine matrix ${ }^{X} C^{Y}$. If a vector $v$ is expressed in the $\mathrm{Y}$ frame as ${ }^{Y} v$, its components in the $\mathrm{X}$ frame are computed by

$$
X_{v}={ }^{X} C^{Y} Y_{v}
$$

Note that ${ }^{Y} C^{X}=\left({ }^{X} C^{Y}\right)^{T}=\left({ }^{X} C^{Y}\right)^{-1}$.

Introducing bases, we obtain

$$
\left[\begin{array}{ccccc}
I_{i} & 0 & 0 & 0 & -\tilde{r}_{i}{ }^{i} C^{N} \\
0 & I_{o} & 0 & 0 & \tilde{r}_{o}{ }^{o} C^{N} \\
0 & 0 & m_{i} \mathbf{1} & 0 & -\mathbf{1} \\
0 & 0 & 0 & m_{o} \mathbf{1} & \mathbf{1} \\
-{ }^{N} C^{i} \tilde{r}_{i}^{T} & { }^{N} C^{o} \tilde{r}_{o}^{T} & -\mathbf{1} & \mathbf{1} & 0
\end{array}\right]\left\{\begin{array}{c}
\dot{\omega}_{i} \\
\dot{\omega}_{o} \\
\dot{v}_{i} \\
\dot{v}_{o} \\
F_{G}
\end{array}\right\}=\left\{\begin{array}{c}
T_{i}-\tilde{\omega}_{i} H_{i}+T_{G} \\
T_{o}-\tilde{\omega}_{o} H_{o}-{ }^{o} C^{i} T_{G} \\
F_{i} \\
F_{o} \\
{ }^{N} C^{i} \bar{\omega}_{i} r_{i}-{ }^{N} C^{o} \bar{\omega}_{o} r_{o}
\end{array}\right\}
$$

This equation is the building block for the multibody case. When assembled, the system equations of motion will be of order $6 N+3(N-1)$, representing three rotational degrees of freedom per body, three translational degrees of freedom per body, and three constraints for each joint. We will see in section IV how to reduce the number of equations to a minimal set.

\section{Generalization to N Bodies}

This section develops the procedure for assembling the system equations of motion using equation (7) as the building block. A tree topology of $N$ bodies has $N-1$ joints. While a body may be associated with multiple joints, each joint has exactly one "inner" body and one "outer" body. Using this as an organizing 
principle allows the assembly of the system equations of motion by inspection. First, we write:

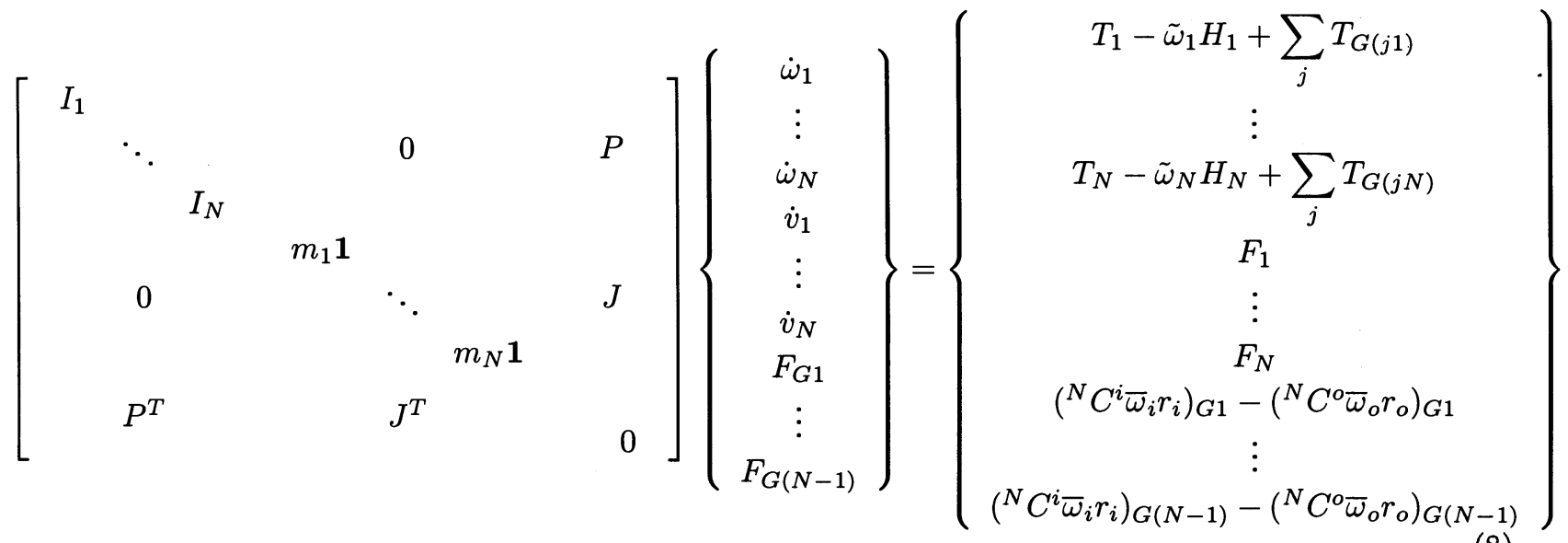

where

$$
T_{G(j k)}=\left\{\begin{array}{cc}
T_{G} & \text { if body } k \text { is } B_{i} \text { for joint } G_{j} \\
-{ }^{o} C^{i} T_{G} & \text { if body } k \text { is } B_{o} \text { for joint } G_{j} \\
0 & \text { otherwise }
\end{array}\right.
$$

The $T_{G(j k)}$ terms are assembled by a simple procedure. For each joint, apply the (user-supplied) joint torque to the joint's inner body, and apply its equal and opposite (properly transformed) to the joint's outer body.

The submatrices $P$ and $J$ share a common structure. Each may be considered as an array of $3 \times 3$ blocks, arranged in $N$ "3-rows" and $N-1$ "3-columns". Each such 3-row corresponds to a body, and each 3-column corresponds to a joint. Thus, each 3 -column contains exactly two nonzero blocks, corresponding to the inner and outer bodies associated with that joint. For each joint $G_{j}$,

$$
\begin{array}{cc}
P_{i j}=-\tilde{r}_{i j}{ }^{i} C^{N}, & P_{o j}=\tilde{r}_{o j}{ }^{o} C^{N} \\
J_{i j}=-\mathbf{1}, & J_{o j}=\mathbf{1}
\end{array}
$$

As an illustrative example, consider the system depicted in figure 3. We construct a tree connectivity table, assigning the inner and outer body for each joint. See table 3.

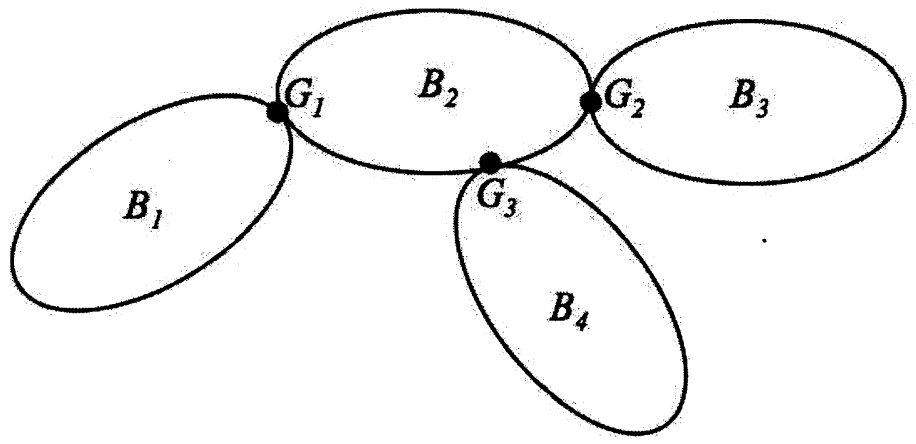

Figure 3. Four-body Example Problem

The submatrices $P$ and $J$ follow the structure of the tree connectivity table:

$$
P=\left[\begin{array}{ccc}
-\tilde{r}_{11}{ }^{1} C^{N} & 0 & 0 \\
\tilde{r}_{21}{ }^{2} C^{N} & -\tilde{r}_{22}{ }^{2} C^{N} & -\tilde{r}_{23}{ }^{2} C^{N} \\
0 & \tilde{r}_{32}{ }^{3} C^{N} & 0 \\
0 & 0 & \tilde{r}_{43}{ }^{4} C^{N}
\end{array}\right], \quad J=\left[\begin{array}{ccc}
-\mathbf{1} & 0 & 0 \\
\mathbf{1} & -\mathbf{1} & \mathbf{- 1} \\
0 & \mathbf{1} & 0 \\
0 & 0 & \mathbf{1}
\end{array}\right]
$$


Table 3. Example Tree Connectivity Table

\begin{tabular}{|c||c|c|c|}
\hline & $G_{1}$ & $G_{2}$ & $G_{3}$ \\
\hline \hline$B_{1}$ & $i$ & & \\
\hline$B_{2}$ & $o$ & $i$ & $i$ \\
\hline$B_{3}$ & & $o$ & \\
\hline$B_{4}$ & & & $o$ \\
\hline
\end{tabular}

The right-hand side of equation (8) is written explicitly as

$$
\left\{\begin{array}{c}
T_{1}-\tilde{\omega}_{1} H_{1}+T_{G 1} \\
T_{2}-\tilde{\omega}_{2} H_{2}-{ }^{2} C^{1} T_{G 1}+T_{G 2}+T_{G 3} \\
T_{3}-\tilde{\omega}_{3} H_{3}-{ }^{3} C^{2} T_{G 2} \\
T_{4}-\tilde{\omega}_{4} H_{4}-{ }^{4} C^{2} T_{G 3} \\
F_{1} \\
F_{2} \\
F_{3} \\
F_{4} \\
{ }^{N} C^{1} \bar{\omega}_{1} r_{11}-{ }^{N} C^{2} \bar{\omega}_{2} r_{21} \\
{ }^{N} C^{2} \bar{\omega}_{2} r_{22}-{ }^{N} C^{3} \bar{\omega}_{3} r_{32} \\
{ }^{N} C^{2} \bar{\omega}_{2} r_{23}-{ }^{N} C^{4} \bar{\omega}_{4} r_{43}
\end{array}\right\}
$$

\section{Reduction of the State Vector}

The equations of motion described in the previous section have a clear structure deriving from their origin in Newton and Euler's equations. This structure facilitates mechanization; the various submatrices may be readily assembled by simple procedures. This formulation suffers, however, from rapid growth of computational effort with the size of the model. The number of equations, $6 N+3(N-1)$, grows three times as fast as the number of degrees of freedom, $3 N+3$. To reduce the computational load, we assume that the joint forces, $F_{G}$, are not of interest, and seek a reduced, minimum-dimension system of $3 N+3$ equations to solve.

We begin by defining a reduced state vector $x$, which has all the desired degrees of freedom, and two other auxiliary vectors $y$ and $f$ :

$$
x=\left\{\begin{array}{c}
\omega_{1} \\
\omega_{2} \\
\vdots \\
\omega_{N} \\
v_{1}
\end{array}\right\}, \quad y=\left\{\begin{array}{c}
v_{2} \\
\vdots \\
v_{N}
\end{array}\right\}, \quad f=\left\{\begin{array}{c}
F_{G 1} \\
\vdots \\
F_{G(N-1)}
\end{array}\right\}
$$

We then repartition equation (8) thus:

$$
\left[\begin{array}{ccc}
A & 0 & R \\
0 & M & U \\
R^{T} & U^{T} & 0
\end{array}\right]\left\{\begin{array}{l}
\dot{x} \\
\dot{y} \\
f
\end{array}\right\}=\left\{\begin{array}{c}
\mathcal{T} \\
\mathcal{F} \\
C
\end{array}\right\}
$$

where the system matrix partitions are correspondingly: 


$$
\begin{gathered}
A=\left[\begin{array}{cccc}
I_{1} & & & \\
& \ddots & & \\
& & I_{N} & \\
& & m_{1} \mathbf{1}
\end{array}\right], \quad M=\left[\begin{array}{ccc}
m_{2} 1 & \\
& \ddots & \\
& & m_{N} \mathbf{1}
\end{array}\right], \\
\mathcal{T}=\left\{\begin{array}{c}
T_{1}-\tilde{\omega}_{1} H_{1}+\sum_{j} T_{G(j 1)} \\
\vdots \\
T_{N}-\tilde{\omega}_{N} H_{N}+\sum_{j} T_{G(j N)} \\
F_{1}
\end{array}\right\}, \mathcal{F}=\left\{\begin{array}{c}
F_{2} \\
\vdots \\
F_{N}
\end{array}\right\}, C=\left\{\begin{array}{c}
\left({ }^{N} C^{i} \bar{\omega}_{i} r_{i}\right)_{G 1}-\left({ }^{N} C^{o} \bar{\omega}_{o} r_{o}\right)_{G 1} \\
\vdots \\
\left({ }^{N} C^{i} \bar{\omega}_{i} r_{i}\right)_{G(N-1)}-\left({ }^{N} C^{o} \bar{\omega}_{o} r_{o}\right)_{G(N-1)}
\end{array}\right\}
\end{gathered}
$$

$R$ is formed by adjoining the first 3-row of $J$ onto $P$, and $U$ is the portion of $J$ that remains. In our four-body example,

$$
R=\left[\begin{array}{ccc}
-\tilde{r}_{11}{ }^{1} C^{N} & 0 & 0 \\
\tilde{r}_{21}{ }^{2} C^{N} & -\tilde{r}_{22}{ }^{2} C^{N} & -\tilde{r}_{23}{ }^{2} C^{N} \\
0 & \tilde{r}_{32}{ }^{3} C^{N} & 0 \\
0 & 0 & \tilde{r}_{43}{ }^{4} C^{N} \\
-\mathbf{1} & 0 & 0
\end{array}\right], \quad U=\left[\begin{array}{ccc}
\mathbf{1} & \mathbf{- 1} & -\mathbf{1} \\
0 & \mathbf{1} & 0 \\
0 & 0 & \mathbf{1}
\end{array}\right]
$$

We now perform row operations to decouple $\dot{x}$ from $\dot{y}$ and $f$. Introducing the unknown coefficient matrices $\alpha$ and $\beta$, we add the three 3 -rows of equation (11):

$$
\left[\left[\begin{array}{ccc}
A & 0 & R
\end{array}\right]+\alpha\left[\begin{array}{lll}
0 & M & U
\end{array}\right]+\beta\left[\begin{array}{lll}
R^{T} & U^{T} & 0
\end{array}\right]\right]\left\{\begin{array}{c}
\dot{x} \\
\dot{y} \\
f
\end{array}\right\}=\mathcal{T}+\alpha \mathcal{F}+\beta C
$$

or

$$
\left[\begin{array}{lll}
A+\beta R^{T} & \alpha M+\beta U^{T} & R+\alpha U
\end{array}\right]\left\{\begin{array}{c}
\dot{x} \\
\dot{y} \\
f
\end{array}\right\}=\mathcal{T}+\alpha \mathcal{F}+\beta C
$$

To decouple $\dot{y}$ and $f$, we solve for $\alpha$ and $\beta$ so that

$$
\begin{aligned}
\alpha M+\beta U^{T} & =0 \\
R+\alpha U & =0
\end{aligned}
$$

from which we find

$$
\begin{aligned}
& \alpha=-R U^{-1} \\
& \beta=R U^{-1} M U^{-T}
\end{aligned}
$$

and the desired form of the equations of motion is

$$
\left(A+\beta R^{T}\right) \dot{x}=\mathcal{T}+\alpha \mathcal{F}+\beta C
$$

This is the system of equations to be solved at each step of the numerical integration. Note that $A, M$, and $U$, (and thus $U^{-1}$ and $U^{-1} M U^{-T}$ ) are constant matrices, and so need only be computed once for a particular system. 


\section{Equations of Motion of Two Bodies with One Gimballed Joint}

To this point, the dynamical formulation assumed spherical joints, possessing three rotational degrees of freedom with no preferred rotation axis. In spacecraft dynamic modelling, applications commonly arise where it is desirable to model a joint having one or two degrees of freedom as a gimballed joint; solar array drives and antenna pointing mechanisms are prime examples. So, in the following sections, we develop equations of motion for a system of rigid bodies linked by gimballed joints, building on the framework already developed for spherical joints.

The rotation of a gimbal joint is described by an Euler sequence of gimbal angles. Considering again the system described by equations (1)-(5), we introduce the gimbal angles, $\theta$, and their associated rates of change, $u \equiv \dot{\theta}$, so that the angular velocity of the outer body may be written as

$$
\omega_{o}=\omega_{i}+\Gamma u
$$

where $\Gamma$ is a 3-by-3 matrix of nonlinear functions of the gimbal angles, depending on the given Euler sequence and whether the rotations are about "space" or "body" axes; see Kane, Likens, and Levinson" for a complete discussion of these terms. For example, a Body-three 1-2-3 Euler rotation through $\theta_{1}, \theta_{2}, \theta_{3}$ has the relationship between ${ }^{i} \omega^{o}$ and $u$ :

$$
\left\{\begin{array}{c}
{ }^{i} \omega_{1}^{o} \\
{ }^{i} \omega_{2}^{o} \\
{ }^{i} \omega_{3}^{o}
\end{array}\right\}=\left[\begin{array}{ccc}
\cos \theta_{2} \cos \theta_{3} & \sin \theta_{3} & 0 \\
-\cos \theta_{2} \sin \theta_{3} & \cos \theta_{3} & 0 \\
\sin \theta_{2} & 0 & 1
\end{array}\right]\left\{\begin{array}{l}
u_{1} \\
u_{2} \\
u_{3}
\end{array}\right\}
$$

Writing it compactly as ${ }^{i} \omega^{o}=\Gamma u$ defines $\Gamma$ for this Euler rotation sequence. Differentiating (14),

$$
\dot{\omega}_{o}=\dot{\omega}_{i}+\Gamma \dot{u}+\dot{\Gamma} u
$$

where $\dot{\Gamma}$ is, like $\Gamma$, a nonlinear function of $\theta$ and $u$. Substituting into (5), some manipulation yields

$$
\dot{v}_{o}=\dot{v}_{i}+\left(\tilde{r}_{o}-\tilde{r}_{i}\right) \dot{\omega}_{i}+\tilde{r}_{o}(\Gamma \dot{u}+\dot{\Gamma} u)+\bar{\omega}_{i} r_{i}-\bar{\omega}_{o} r_{o}
$$

and the equations of motion for the two-body system are

$$
\left[\begin{array}{cccc}
I_{i} & 0 & 0 & -\tilde{r}_{i} \\
I_{o} & I_{o} \Gamma & 0 & \tilde{r}_{o} \\
0 & 0 & m_{i} \mathbf{1} & -\mathbf{1} \\
m_{o}\left(\tilde{r}_{o}-\tilde{r}_{i}\right) & m_{o} \tilde{r}_{o} \Gamma & m_{o} \mathbf{1} & \mathbf{1}
\end{array}\right]\left\{\begin{array}{c}
\dot{\omega}_{i} \\
\dot{u} \\
\dot{v}_{i} \\
F_{G}
\end{array}\right\}=\left\{\begin{array}{c}
T_{i}-\tilde{\omega}_{i} H_{i}+T_{G} \\
T_{o}-\tilde{\omega}_{o} H_{o}-T_{G}-I_{o} \dot{\Gamma} u \\
F_{i} \\
F_{o}-m_{o} \tilde{r}_{o} \dot{\Gamma} u+m_{o}\left(\bar{\omega}_{i} r_{i}-\bar{\omega}_{o} r_{o}\right)
\end{array}\right\}
$$

Comparing with equation (6), we see some familiar patterns, but not enough to extrapolate with confidence to the N-body problem. For further illumination, we turn to the four-body example of figure 3 . The equations of motion for this example may be written

$$
\left[\begin{array}{ccc}
I & 0 & P \\
\Pi & \mu & J
\end{array}\right]\left\{\left\{\begin{array}{c}
\dot{\omega}_{1} \\
\dot{u}_{1} \\
\dot{u}_{2} \\
\dot{u}_{3} \\
\dot{v}_{1} \\
F_{G}
\end{array}\right\}=\left\{\begin{array}{c}
\mathcal{T} \\
\mathcal{F}
\end{array}\right\}\right.
$$

with $P$ and $J$ as defined in equation (10) except that we have not yet introduced coordinate bases, and

$$
I=\left[\begin{array}{cccc}
I_{1} & 0 & 0 & 0 \\
I_{2} & I_{2} \Gamma_{1} & 0 & 0 \\
I_{3} & I_{3} \Gamma_{1} & I_{3} \Gamma_{2} & 0 \\
I_{4} & I_{4} \Gamma_{1} & 0 & I_{4} \Gamma_{3}
\end{array}\right], \quad \mu=\left[\begin{array}{c}
m_{1} \mathbf{1} \\
m_{2} \mathbf{1} \\
m_{3} \mathbf{1} \\
m_{4} \mathbf{1}
\end{array}\right]
$$




$$
\begin{aligned}
& \Pi=\left[\begin{array}{cccc}
0 & 0 & 0 & 0 \\
m_{2}\left(\tilde{r}_{21}-\tilde{r}_{11}\right) & m_{2} \tilde{r}_{21} \Gamma_{1} & 0 & 0 \\
m_{3}\left(\tilde{r}_{32}-\tilde{r}_{22}+\tilde{r}_{21}-\tilde{r}_{11}\right) & m_{3}\left(\tilde{r}_{32}-\tilde{r}_{22}+\tilde{r}_{21}\right) \Gamma_{1} & m_{3} \tilde{r}_{32} \Gamma_{2} & 0 \\
m_{4}\left(\tilde{r}_{43}-\tilde{r}_{23}+\tilde{r}_{21}-\tilde{r}_{11}\right) & m_{4}\left(\tilde{r}_{43}-\tilde{r}_{23}+\tilde{r}_{21}\right) \Gamma_{1} & 0 & m_{4} \tilde{r}_{43} \Gamma_{3}
\end{array}\right] \\
& \mathcal{T}=\left\{\begin{array}{c}
T_{1}-\tilde{\omega}_{1} H_{1}+T_{G 1} \\
T_{2}-\tilde{\omega}_{2} H_{2}-T_{G 1}+T_{G 2}+T_{G 3}-I_{2} \dot{\Gamma}_{1} u_{1} \\
T_{3}-\tilde{\omega}_{3} H_{3}-T_{G 2}-I_{3}\left(\dot{\Gamma}_{1} u_{1}+\dot{\Gamma}_{2} u_{2}\right) \\
T_{4}-\tilde{\omega}_{4} H_{4}-T_{G 3}-I_{4}\left(\dot{\Gamma}_{1} u_{1}+\dot{\Gamma}_{3} u_{3}\right)
\end{array}\right\} \\
& \mathcal{F}=\left\{\begin{array}{c}
F_{1} \\
F_{2}-m_{2} \tilde{r}_{21} \dot{\Gamma}_{1} u_{1}+m_{2}\left(\bar{\omega}_{1} r_{11}-\bar{\omega}_{2} r_{21}\right) \\
F_{3}-m_{3}\left(\tilde{r}_{32}-\tilde{r}_{22}+\tilde{r}_{21}\right) \dot{\Gamma}_{1} u_{1}-m_{3} \tilde{r}_{32} \dot{\Gamma}_{2} u_{2}+m_{3}\left(\bar{\omega}_{1} r_{11}-\bar{\omega}_{2} r_{21}\right)+m_{3}\left(\bar{\omega}_{2} r_{22}-\bar{\omega}_{3} r_{32}\right) \\
F_{4}-m_{4}\left(\tilde{r}_{43}-\tilde{r}_{23}+\tilde{r}_{21}\right) \dot{\Gamma}_{1} u_{1}-m_{4} \tilde{r}_{43} \dot{\Gamma}_{3} u_{3}+m_{4}\left(\bar{\omega}_{1} r_{11}-\bar{\omega}_{2} r_{21}\right)+m_{4}\left(\bar{\omega}_{2} r_{23}-\bar{\omega}_{4} r_{43}\right)
\end{array}\right\}
\end{aligned}
$$

While some of the symmetries of the spherical joint system are lost, new patterns emerge. The $I$ and $\Pi$ matrices have a common structure stemming from the composition of angular velocities by concatenation of gimbal rates with $\omega_{1}$. For a system of $N$ bodies, the matrix $I$ is $3 N \times 3 N$, each 3-row $j$ corresponding to a body, and each 3-column $k$ corresponding to a point. The first 3-column corresponds to the mass center of $B_{1}$, since it multiplies $\dot{\omega}_{1}$. Each succeeding 3-column corresponds to a joint. Each 3-row may be assembled by inspection, by traversing the tree from $B_{1}$ to $B_{j}$, placing an $I_{j} \Gamma_{k-1}$ term for each joint in the path. Similarly, the elements of the $\Pi$ matrix are parallel-axis (" $m r^{2}$ ") terms, the $r$ vector being the vector from the mass center of $B_{j}$ to point $k$. The novel terms in $\mathcal{T}$ and $\mathcal{F}$ may be likewise constructed; compare $I$, $\Pi, \mathcal{T}$, and $\mathcal{F}$ with the path traversals tabulated in table 4 . These patterns will lead us to codify rules for assembly of equations of motion for a general system. First we must introduce coordinate bases.

Table 4. Example Tree Path Table

\begin{tabular}{|c||c|c|c|}
\hline & $G_{1}$ & $G_{2}$ & $G_{3}$ \\
\hline \hline$B_{1}$ & & & \\
\hline$B_{2}$ & $\times$ & & \\
\hline$B_{3}$ & $\times$ & $\times$ & \\
\hline$B_{4}$ & $\times$ & & $\times$ \\
\hline
\end{tabular}

Assigning bases proceeds as with the spherical joints, except that $\Gamma \dot{u}$ and $\dot{\Gamma} u$ terms are naturally expressed in the inner body frame for "space" sequences, but in the outer body for "body" sequences. Subsequent discussion assumes a body sequence.

Considering again the two-body case, we observe that it is most convenient to express the first equation in $B_{i}$, the second in $B_{o}$, and the remaining two in the $N$ frame. Inserting the appropriate direction cosine matrices, equation (15) becomes

$$
\left.\begin{array}{c}
{\left[\begin{array}{cccc}
I_{i} & 0 & 0 & -\tilde{r}_{i}{ }^{i} C^{N} \\
I_{o} & I_{o} \Gamma & 0 & \tilde{r}_{o}{ }^{o} C^{N} \\
0 & 0 & m_{i} \mathbf{1} & -\mathbf{1} \\
m_{o}\left({ }^{N} C^{o} \tilde{r}_{o}-{ }^{N} C^{i} \tilde{r}_{i}\right) & m_{o}{ }^{N} C^{o} \tilde{r}_{o} \Gamma & m_{o} \mathbf{1} & \mathbf{1}
\end{array}\right]\left\{\begin{array}{c}
\dot{\omega}_{i} \\
\dot{u} \\
\dot{v}_{i} \\
F_{G}
\end{array}\right\}=} \\
T_{i}-\tilde{\omega}_{i} H_{i}+T_{G} \\
T_{o}-\tilde{\omega}_{o} H_{o}-{ }^{o} C^{i} T_{G}-I_{o} \dot{\Gamma} u \\
F_{i} \\
F_{o}-m_{o}{ }^{N} C^{o} \tilde{r}_{o} \dot{\Gamma} u+m_{o}\left({ }^{N} C^{i} \bar{\omega}_{i} r_{i}-{ }^{N} C^{o} \bar{\omega}_{o} r_{o}\right)
\end{array}\right\}
$$

The equations of motion for the assembled system are still equation (16). For notational convenience, we consider the mass center of $B_{1}$ to be the zeroth joint, $G_{0}$, so that $\Gamma_{0} \equiv \mathbf{1}$ and $\left(\tilde{r_{o}}\right)_{0} \equiv \mathbf{0}$. Then the system 
submatrices may be written:

$$
\begin{gathered}
I_{j k}=\left\{\begin{array}{cl}
I_{j} \Gamma_{k-1} & \text { if } G_{k-1} \text { is in path from } B_{1} \text { to } B_{j} \quad j, k=1, \ldots, N \\
0 & \text { otherwise }
\end{array}\right. \\
\Pi_{j k}=m_{j}\left\{\left({ }^{N} C^{o} \tilde{r_{o}}\right)_{k-1}+\sum_{l}\left({ }^{N} C^{o} \tilde{r}_{o}-{ }^{N} C^{i}{\tilde{r_{i}}}^{i} C^{o}\right)_{l}\right\} \Gamma_{k-1} \quad \begin{array}{l}
j, k=1, \ldots, N \\
l: \text { All } G \text { in path between } G_{k-1} \text { and } B_{j}
\end{array} \\
\mu_{j}=m_{j} 1, \quad j=1, \ldots, N
\end{gathered}
$$

$P$ and $J$ are defined as in equation (10).

$$
\begin{gathered}
\mathcal{T}_{j}=T_{j}-\tilde{\omega}_{j} H_{j}+\sum_{k} T_{G(j k)}-I_{j} \sum_{l}{ }^{j} C^{l} \dot{\Gamma}_{l} u_{l} \quad \begin{array}{l}
j, k=1, \ldots, N \\
T_{G} \text { defined in equation (9) } \\
l: \text { All } G \text { in path from } B_{1} \text { to } B_{j}
\end{array} \\
\mathcal{F}_{j}=F_{j}-m_{j}\left\{\sum_{k}\left[\left({ }^{N} C^{o} \tilde{r}_{o}\right)_{k}+\sum_{l}\left({ }^{N} C^{o} \tilde{r_{o}}-{ }^{N} C^{i}{\tilde{r_{i}}}^{i} C^{o}\right)_{l}\right] \dot{\Gamma}_{k} u_{k}+\sum_{k}\left[{ }^{N} C^{o} \bar{\omega}_{o} r_{o}-{ }^{N} C^{i} \bar{\omega}_{i} r_{i}\right]_{k}\right\} \\
j=1, \ldots, N \\
k: \text { All } G \text { in path from } B_{1} \text { to } B_{j} \\
l: \text { All } G \text { in path between } G_{k} \text { and } B_{j}
\end{gathered}
$$

\section{Elimination of Constraint Forces and Constrained Gimbal Degrees of Freedom}

Recall from section IV that the spherical joint formulation led to a system of dimension $9 N-3$, which we then reduced to a $3 N+3$ system by row operations. The gimballed joint formulation starts from a more advantageous position, in that the redundant velocity and angular velocity states have already been reduced by combination into the gimbal angular rates, $u$, resulting in a system of dimension $6 N$. To arrive at a $3 N+3$ system, we eliminate the $3 N-3$ joint forces, $F_{G}$, by row operations. Further, the motivation for pursuing the gimballed formulation is to eliminate undesired degrees of freedom, such as the two constrained axes of a single-axis solar array drive mechanism, from the final equations of motion. With this formulation, these degrees of freedom are easily eliminated.

First we eliminate the joint constraint forces by defining the state vector as

$$
x=\left\{\begin{array}{c}
\omega_{1} \\
u \\
v_{1}
\end{array}\right\}
$$

and partitioning the assembled equations of motion as

$$
\left[\begin{array}{cc}
A & R \\
S & U
\end{array}\right]\left\{\begin{array}{c}
\dot{x} \\
F_{G}
\end{array}\right\}=\left\{\begin{array}{c}
\mathcal{T} \\
\mathcal{F}
\end{array}\right\}
$$

The subsystem matrix $A$ is formed by adjoining the first 3-rows of $\Pi$ and $\mu$ to the $\left[\begin{array}{ll}I & 0\end{array}\right]$ of equation (16):

$$
A=\left[\begin{array}{cc}
I & 0 \\
\Pi_{1} & \mu_{1}
\end{array}\right]
$$

and $S$ comprises the remainders of $\Pi$ and $\mu$ :

$$
S=\left[\begin{array}{ll}
\Pi_{2, \ldots, N} & \mu_{2, \ldots, N}
\end{array}\right]
$$


As for the spherical-joint case, the submatrix $R$ is formed by adjoining the first 3-row of $J$ onto $P$, and $U$ is the portion of $J$ that remains. Again using an unknown coefficient matrix, $\alpha$, so that

$$
\begin{array}{rll}
(A+\alpha S) \dot{x} & = & \mathcal{T}+\alpha \mathcal{F} \\
R+\alpha U & = & 0
\end{array}
$$

we solve for $\alpha$,

$$
\alpha=-R U^{-1}
$$

Substituting, we obtain the reduced equations of motion,

$$
\left(A-R U^{-1} S\right) \dot{x}=\mathcal{T}-R U^{-1} \mathcal{F}
$$

Finally, we consider the gimbal degrees of freedom which are to be eliminated due to their being constrained or negligible. In the spherical joint case, these degrees of freedom were complicated combinations of the states. With the dynamics reformulated in terms of gimbal states, eliminating undesired degrees of freedom is as straightforward as striking out rows and columns of the system matrices. To make this clear, expand the state vector into its scalar components,

$$
x=\left\{\begin{array}{llllllllll}
\omega_{11} & \omega_{12} & \omega_{13} & u_{11} & u_{12} & u_{13} & \cdots & u_{N 1} & u_{N 2} & u_{N 3}
\end{array}\right\}
$$

where $u_{j k}$ is associated with the $k$ th degree of freedom of the $j$ th joint. Suppose, as an example, that joint 1 is a two-axis gimbal, so that $u_{13}=\dot{u}_{13}=0$. Looking at the system of equation (18), the sixth column of $\left(A-R U^{-1} S\right)$ multiplies zero, and the sixth row becomes a constraint equation on the reaction torque at the gimbal. Eliminating the sixth row and column of the system removes the undesired degree of freedom, reducing the system order by one. In the implementation, this state reduction may be accomplished during the assembly of $A, R, S$, and $\mathcal{T}$.

\section{Some Simulation Results}

As a demonstration of the methods developed in this paper, some early MMS simulation results are presented. As has been discussed, the interaction of spin, boom flexibility, and thruster activity provides some interesting problems. A thorough discussion of these is beyond the scope of this paper; our purpose here is simply to show that the numerical methods have been successfully implemented.

Three spacecraft models are implemented. Each consists of a central body with four "wire" appendages. For the first spacecraft, each appendage is modeled as a single rigid body. The second and third spacecraft model each appendage as two and three connected rigid bodies, respectively. Spherical joints are used, with joint torques consisting of light damping provided through a user-supplied function. The numerical integration of the dynamics is accomplished using the same software functions for all three models. The body and joint information that defines each model is input from text files and stored in assigned data structures, and the dynamic system matrices are assembled as needed at each simulation timestep. All three spacecraft models are simulated concurrently.

The booms are given small initial deflections to excite both in-plane and out-of-plane oscillations. Figure 4 shows the motion of one boom tip, for all three models. All models show the interaction between in-plane and out-of-plane boom dynamics, coupled through spacecraft spin. The second and third modes of the boom are most evident in the initial transient of the in-plane tip motion of the two- and three-segment models. These higher-frequency modes are attenuated by the damping torques in the joints. The model comparison lends confidence both that the important dynamic features have been captured in the model, and that the mathematical models have been correctly implemented in software.

\section{Conclusion}

Beginning from Newton's and Euler's equations of motion for a single rigid body, we have presented a method for constructing the dynamic equations of motion of a general system of rigid bodies connected by spherical or gimballed joints in a tree topology. Two guiding ideas make this a tractable problem. First, each joint in a tree topology has exactly one "inner" body and one "outer" body. This allows a system of arbitrary 

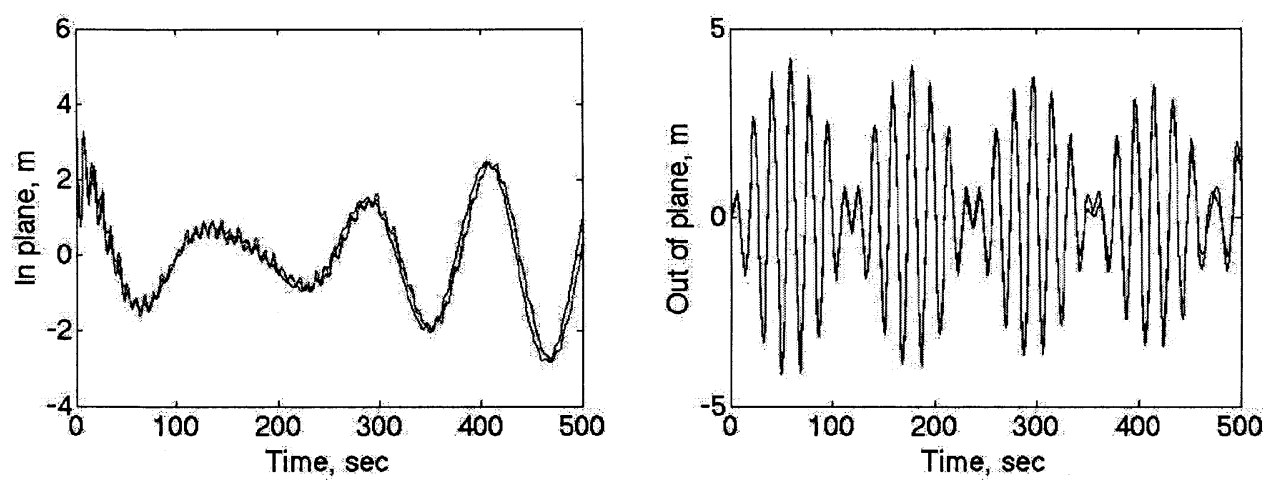

Figure 4. Wire Boom Tip Motion

complexity to be assembled from the equations of motion of a two-body system. The assembly procedure is easy to implement in software, and relatively easy to check by inspection of the various arrays as they are populated. The second idea is that elimination of constraint equations may be considered as a problem in linear algebra to be solved numerically at each timestep, rather than a dynamics problem to be solved during formulation. For simple systems, it may be practical to symbolically eliminate the $3 N-3$ constraint equations, yielding some analytical insight. This rapidly becomes impractical, however, as the system grows in complexity. The assembly method presented here allows straightforward verification that the matrices $A, M$, etc. have been properly loaded into memory, as the patterns and symmetries in the building blocks are not obscured. Elimination of the constraint equations is then a purely mechanical process of matrix inversions and multiplication.

\section{Appendix: Auxiliary Equations of Motion}

\section{Kinematic Equations of Motion}

The main body of this paper discusses the dynamic equations of motion, which are solved to find the dynamic state variables, $\omega$ and $v$. For completeness, we present without derivation the additional equations of motion associated with the kinematic state variables: the quaternion, $q$, representing attitude, and the position vector, $p$.

Defining the quaternion elements in terms of a simple rotation about an axis $\hat{\lambda}$ through an angle $\theta$ :

$$
\left\{\begin{array}{l}
q_{1} \\
q_{2} \\
q_{3} \\
q_{4}
\end{array}\right\}=\left\{\begin{array}{c}
\lambda_{1} \sin (\theta / 2) \\
\lambda_{2} \sin (\theta / 2) \\
\lambda_{3} \sin (\theta / 2) \\
\cos (\theta / 2)
\end{array}\right\}
$$

the time derivatives of the quaternion elements are functions of the quaternion and the angular velocity:

$$
\left\{\begin{array}{c}
\dot{q}_{1} \\
\dot{q}_{2} \\
\dot{q}_{3} \\
\dot{q}_{4}
\end{array}\right\}=\frac{1}{2}\left[\begin{array}{ccc}
q_{4} & -q_{3} & q_{2} \\
q_{3} & q_{4} & -q_{1} \\
-q_{2} & q_{1} & q_{4} \\
-q_{1} & -q_{2} & -q_{3}
\end{array}\right]\left\{\begin{array}{l}
\omega_{1} \\
\omega_{2} \\
\omega_{3}
\end{array}\right\}
$$

The translational kinematic equations of motion are simply:

$$
\dot{p}=v
$$

\section{Equations of Motion for Embedded Momentum Wheels}

For a single rigid body $B$, Euler's equation is written:

$$
I \dot{\omega}=T-\omega \times H
$$


where $H=I \omega$. We consider here the case of one or more momentum wheels embedded in a rigid body. Consider a reaction wheel, $W$, having rotational inertia $J$, scalar angular momentum $h_{w}$, and whose axis, $\hat{a}$, is fixed in $B$. Momentum is exchanged between $B$ and $W$ by applying equal and opposite interaction torques (typically specified by some control law). We denote this interaction torque as a scalar, $T_{w}$; we apply it (arbitrarily) in a positive sense to the wheel, and its opposite to $B$. The modified equations of motion are:

$$
\begin{gathered}
I \dot{\omega}=T-\omega \times\left(I \omega+h_{w} \hat{a}\right)-T_{w} \hat{a} \\
\dot{h}_{w}=T_{w}-J \hat{a} \cdot \dot{\omega}
\end{gathered}
$$

Multiple wheels act in superposition:

$$
\begin{gathered}
I \dot{\omega}=T-\omega \times\left(I \omega+\sum_{k=1}^{n} h_{w k} \hat{a}_{k}\right)-\sum_{k=1}^{n} T_{w k} \hat{a}_{k} \\
\dot{h}_{w k}=T_{w k}-J_{k} \hat{a}_{k} \cdot \dot{\omega}, \quad(k=1,2, \ldots, n)
\end{gathered}
$$

\section{References}

\footnotetext{
${ }^{1}$ Kane, Thomas R., Likins, Peter W., Levinson, David A. Spacecraft Dynamics. McGraw-Hill Book Company, 1983.

${ }^{2}$ Hughes, Peter C. Spacecraft Attitude Dynamics. John Wiley \& Sons, 1986.

${ }^{3}$ Wertz, James R. (ed). Spacecraft Attitude Determination and Control. Kluwer Academic Publishers, 1978.

${ }^{4}$ Wie, Bong. Space Vehicle Dynamics and Control. AIAA Education Series, 1998.
} 